\title{
The Analysis of Alpha-1-Antitrypsin Glycosylation with Direct LC-
}

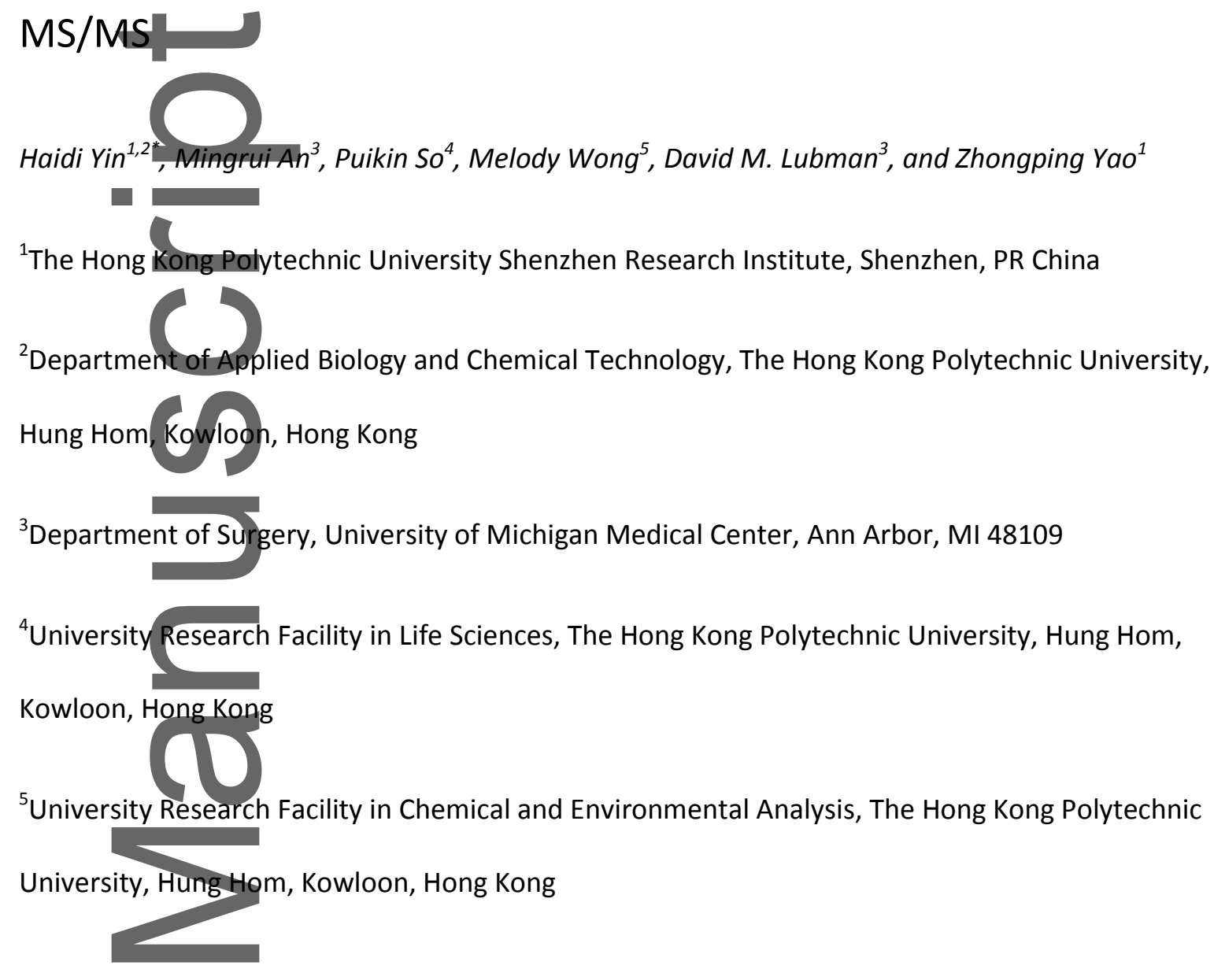

\section{Author Email Address}

Haidi Yin haidi.yin@polyu.edu.hk

Mingrui An_mingruia@umich.edu

Puikin So pui-kin.so@polyu.edu.hk

MelodyWong ym-melody.wong@polyu.edu.hk

David M. Lubman dmlubman@umich.edu

This is the al 1 manuscript accepted for publication and has undergone full peer review but has not been the wh tl copyediting, typesetting, pagination and proofreading process, which may lead to differences betrm this version and the Version of Record. Please cite this article as doi: 10.1002/elps.201700426.

This article is protected by copyright. All rights reserved. 
Running Title: The glycopeptide analysis of A1AT

* To whom correspondence should be addressed. Haidi Yin, The Hong Kong Polytechnic University Shenzhen Research Institute, Shenzhen, PR China; Department of Applied Biology and Chemical Technology,W704, The Hong Kong Polytechnic University, Hung Hom, Kowloon, Hong Kong. E-mail: haidi.yin@polyu.edu.hk; Phone: 0852-34008808

Abbreviations: A1AT, alpha-1-antitrypsin; LC-MS/MS, liquid chromatography with tandem mass spectrometry; TCEP, tris (2-carboxyethyl) phosphine, IAA, iodoacetamide; TEAB, Triethylammonium bicarbonate; TFA, trifluoroacetic acid; CE, collision energy; XIC, Extracting lon Current; CID, collisioninduced dissociation; HCD, high energy collision dissociation

Keywords: alpha-1-antitrypsin, in-source collision-induced dissociation, mass spectrometry, fucosylation

Total Number of Words: $\mathbf{5 , 4 5 8}$
Abstract
A liquid chromatography-tandem mass spectrometry (LC-MS/MS)-based methodology has been developed to differentiate core- and antennary-fucosylated glycosylation of glycopeptides. Both the glycosylation sites (heterogeneity) and multiple possible glycan occupancy at each site (microheterogeneity) can be resolved via intact glycopeptide analysis. The serum glycoprotein alpha- 1-antitrypsin (A1AT) which contains both core- and antennary-fucosylated glycosites was used in this study. Sialidase was used to remove the sialic acids in order to simplify the glycosylation microheterogeneity and to enhance the MS signal of glycopeptides with similar glycan structures. ß1-3,4 galactosidase was used to differentiate core- and antennary-fucosylation. In-source dissociation was found to severely affect the identification and quantification of glycopeptides with 
low abundance glycan modification. The settings of the mass spectrometer were therefore optimized to minimize the in-source dissociation. A three-step mass spectrometry fragmentation strategy was used for glycopeptide identification, facilitated by pGlyco software annotation and manual checking. The collision energy used for initial glycopeptide fragmentation was found to be crucial for improved detection of oxonium ions and better selection of Y1 ion (peptide+GlcNAc). Structural assignments revealed that all 3 glycosylation sites of A1AT glycopeptides contain complex N-glycan structures: site Asn70 contains biantennary glycans without fucosylation; site Asn107 contains bi-, tri-and tetra-antennary glycans with both core- and antennary-fucosylation; site Asn271 contains bi- and tri-antennary glycans with both core- and antennary-fucosylation. The relative intensity of core- and antennary-fucosylation on Asn107 was similar to that of the A1AT protein indicating that the glycosylation level of Asn107 is much larger than the other 2 sites. 1 Introduction Aberrant protein glycosylation especially fucosylation has been found to be associated with various diseases such as cancers[1]. The fucose that attaches to core $\mathrm{N}$-acetylglucosamine of $\mathrm{N}$-glycans is core-fucosylation and those that attach to the antennary $\mathrm{N}$-acetylglucosamine or galactose is antennary fucosylation. The change in core- or antennary-fucosylation of some proteins has been found to be indicative for various cancers. For example, the enhanced level of the core-fucosylation of alpha-fetoprotein (AFP-L3) in the serum was found to be associated with hepatocellular carcinoma[2]. AFP-L3 is detected using a Lens culinaris lectin (LCA) blot assay based on immunoassay and the high affinity of LCA to core-fucosylated glycoproteins[3]. Another example is CA19-9, a type of antennary-fucosylation sialyl lewis A structure. The enhanced level of CA19-9 in the serum is the most widely used clinical marker for pancreatic cancer[4]. CA19-9 is monitored by immunoassay using a sialyl lewis A structure specific antibody[4]. This method relies on a specific antibody so it cannot be easily applied to other glycoproteins. In addition to the above immunoassay based method, another conventional approach for core- and antennary-fucosylation analysis involves 
a combination of various fucosidases and several cycles of HPLC separation[5] of glycans after cleaving glycans from glycoproteins. Although a recently developed immobilized PNGase F digestion procedure has enabled fast release of glycans from glycoproteins[6], the approach of fucosidase digestion is tedious. More importantly, most proteins have multiple fucosylation sites where the above analyses lose the site-specific information and thus cannot provide direct evidence for core- or antennary- fucosylation aberration of proteins, which is key for precise diagnosis.

Many studies have been exploring MS-based profiling of intact glycopeptides, such as increasing sensitivity, resolution and fragmentation of mass spectrometers and developing software for the data analysis of glycopeptides[7, 8]. Studies have been using CID, ECD, ETD, EThcD, low and high energy $\mathrm{HCD}$ fragmentation et. al. or combinations of these fragmentation methods[9-11] to elucidate the structure of glycopeptides. Several different softwares for elucidating these spectra have been developed, among which Byonics[12] and GPQuest[13] are so far the most widely used. However, Byonics relies on peptide sequence-based scoring which underestimates the false positive (

discovery rate of glycopeptides[14] and GPQuest needs a sample-originated peptide library for matching of glycopepties, which makes the experiment more complicated [13]. Here we employed the newly developed pGlyco software from the groups of Yang PY and He SM to facilitate the MS analysis of glycopeptides. pGlyco uses HCD MS2 generated oxonium ions to filter glycopeptides, uses HCD MS3 on Y1 ions for peptide sequencing, and uses CID MS2 for glycan elucidation[15]. pGlyco2.0 is an updated version, which uses stepped HCD collision[14]. Although the involvement of MS3 in peptide sequences with much more complex fragments compared with pGlyco2.0. We therefore used pGlyco as the preferred method.

Using LC-MS/MS alone, it is often difficult to distinguish core- and antennary-fucosylation due to their similar retention time on a C18 column and the same $\mathrm{m} / \mathrm{z}$ of the glycopeptides[16] and then there is also possible migration of fucose from antennary- to core-position during MS/MS 
fragmentation[17]. Glycan derivatization such as permethylation is able to solve the problem of fucose migration, but before derivatization glycans need to be released from glycopeptides so that the site-specific information is lost $[17,18]$. We thus sought to develop a method to differentiate coreand antennary-fucosylation prior to LC-MS/MS and to use pGlyco facilitated mass spectrometry analysis to identify and semi-quantify core- and antennary-fucosylation. In this study, we applied sialidase and galactosidase double digestion to differentiate core- and antennary-fucosylation before mass spectrometry analysis. Sialidase was used to remove sialic acids to simplify the glycosylation microheterogeneity and to enhance the MS signal of glycopeptides. ß1-3,4 galactosidase (from bovine testis) was used to differentiate core- and antennary- fucosylation, where galactosidase is not able to cleave galactose from antennary fucosylated Lewis structures[19].

The fucosylation level of serum protein alpha-1-antitrypsin (A1AT) has been identified as a potential biomarker for various cancers[20,21] and inflammation[22]. In this study, sialidase and galactosidase double digestion of glycopeptide was followed by direct LC-MS/MS analysis without cleaving glycans from the glycopeptides. Both glycosylation site and multiple possible glycan occupancy at each site were resolved, with successful identification and semi-quantification of the glycopeptides of A1AT and with clear differentiation of core- and antennary-fucosylation.

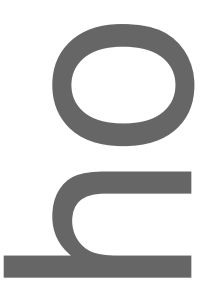

\section{Materials and Methods}

2.1 Trypsin Digestion of protein into peptides: We added $10 \mu \mathrm{L}$ of $50 \mathrm{mM}$ ammonia bicarbonate to $10 \mu \mathrm{g}$ alpha-1-antitrypsin (A1AT) and pipetted to dissolve the sample well. The dissolved A1AT was reduced with $10 \mathrm{mM}$ tris (2-carboxyethyl) phosphine (TCEP) at $37^{\circ} \mathrm{C}$ for $30 \mathrm{~min}$ and alkylated with 20 $\mathrm{mM}$ iodoacetamide (IAA) at room temperature in the dark for $15 \mathrm{~min}$. The sample solution is diluted for 3 times with $50 \mathrm{mM}$ ammonia bicarbonate and incubated with $1 \mu \mathrm{L}$ of $0.5 \mu \mathrm{g} / \mu \mathrm{L}$ trypsin 
(Promega, Madison, $\mathrm{WI}$ ) at $37^{\circ} \mathrm{C}$ for $16 \mathrm{~h}$. The trypsin is eventually deactivated at $95^{\circ} \mathrm{C}$ for $5 \mathrm{~min}$ and dried in a speedvac.

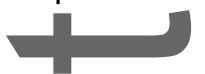

2.2 Enrichment and buffer-exchange of glycopeptides: 3K Ultra centrifugal filter-15 (Millipore Amicon) was used for glycopeptide enrichment and for buffer exchange. The buffer system was changed from the above system to $25 \mathrm{mM}$ sodium acetate (pH5.5) for 3 times at 7,500 $\mathrm{g}$ for $1 \mathrm{~h}$. Glycopeptides with modification were larger than $3 \mathrm{~K}$ so that only non-glycopeptides smaller than $3 \mathrm{~K}$ will pass through the $3 \mathrm{~K}$ membrane.

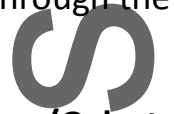

2.3 Sialidase/Galactosidase Double Digestion: For the sialidase and galactosidase digestion, the glycopeptide mixture in $30 \mu \mathrm{L}$ of $25 \mathrm{mM}$ sodium acetate solution was incubated with $15 \mathrm{mU}(3 \mu \mathrm{L})$ of non-specific $\alpha 2-3,6,8,9$ sialidase recombinant from Arthrobacter ureafaciens expressed in E.coli (Prozyme, Hayward, CA) and $75 \mathrm{mU}(3 \mu \mathrm{L})$ of $\beta 1-3,4$ galactosidase from bovine testis (Prozyme, Hayward, CA) at $37^{\circ} \mathrm{C}$ for $18 \mathrm{~h}$ to remove all sialic acid residues and galactose provided that no fucose is bound to the sub-terminal $\mathrm{N}$-acetylglucosamine in an $\mathrm{N}$-glycan. The glycosidases were deactivated at $95^{\circ} \mathrm{C}$ for $5 \mathrm{~min}$.

2.4 C18 Desalting: Trifluoroacetic acid (TFA) was added until the $\mathrm{pH}$ value reached 2. The C18 columns (Fisher Scientific, San Jose, CA) were activated with $200 \mu \mathrm{L} 0.1 \%$ TFA in 50\% acetonitrile for 5 times and equilibrated with $0.1 \%$ TFA in water for 3 times by centrifugation at 1,500 g/min for 1 min each time. The peptides were bound to the $\mathrm{C} 18$ beads for 5 times followed by 3 times washing with $0.1 \%$ TFA to remove non-specific binding by centrifugation as described above; $20 \mu \mathrm{L}$ of $50 \%$ acetonitrile with 0.1\% TFA was used for elution by centrifugation as described above. Elution was repeated once and the combined eluents were then dried in a speedvac.

\subsection{LC-MSIdentification of Glycopeptides}

Nano LC-MS/MS conditions were as described in previous work[23]. A C18 capillary column (100 $\mu \mathrm{m}$ $\times 15 \mathrm{~cm} ; 3 \mu \mathrm{m}$ particles, $200 \AA$ ) (Thermo fisher Scientific, San Jose, CA) was used for LC separation, 
and gradient elution was performed using an Ultimate 3000 nanoLC system (Thermo fisher Scientific, San Jose, CA) with a flow rate of $350 \mathrm{~nL} / \mathrm{min}$. The mobile phase A was $2 \%$ acetonitrile with $0.1 \%$ CA)

formic acid in water and mobile phase B was $2 \%$ water with $0.1 \%$ formic acid in acetonitrile. The analytical gradient lasted for $100 \mathrm{~min}$ where after $10 \mathrm{~min}$ balancing time, the composition of solvent B rose from $3 \%$ to $7 \%$ in $2 \mathrm{~min}$, from $7 \%$ to $14 \%$ in $8 \mathrm{~min}$, from $14 \%$ to $25 \%$ in $55 \mathrm{~min}$, followed by a washing and equilibration step where solvent B increased to $90 \%$ in $5 \mathrm{~min}$ and was held for $8 \mathrm{~min}$, and then returned to $3 \% \mathrm{~B}$ in $0.1 \mathrm{~min}$ and was held for $17 \mathrm{~min}$.

An Orbitrap Fusion Lumos mass spectrometer (Thermo Fisher Scientific, San Jose, CA) operated in positive ion mode was used for analysis. The ESI spray voltage and capillary voltage were set as described in the following part. Two runs of LC-MS were performed for each sample. Each run has two consecutive MS scan types. In the first run, glycopeptides were selected by the detection of oxonium ion 138.05 with low energy HCD MS2; consequently the $\mathrm{Y} 1$ ion (peptide+GlcNAc) from the glycopeptide fragment was subjected to high energy HCD MS3 for peptide sequencing. In the second run, after glycopeptide selection by low energy HCD MS2, the selected glycopeptide was subjected to CID MS2 for glycan structure analysis. The collision energy for each step of fragmentation was also optimized for better detection of oxonium ions, better selection of $\mathrm{Y} 1$ ion and better fragmentation of $\mathrm{Y} 1$ ion and glycans, as discussed in the results section. A full scan defines the mass range of $\mathrm{m} / \mathrm{z}$ 600 to 1800 , and MS/MS was performed with top speed mode.

\subsection{Database Search for Glycopeptide Identification:}

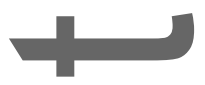

The searchengines.pGlyco and pFind developed by He SM's group were used for glycoprotein analysis. The raw data of two LC-MS runs were aligned first to make sure the retention time of the same precursor ion was the same in the two runs. pFind was used for $\mathrm{Y} 1$ peptide identification using MS3 spectra from the first LC-MS run: (1) fixed modification: cysteine carbamidomethylation (+57.021 Da); (2) Dynamic modification: methionine oxidation (+15.995 Da) and NexHAc (+203.075 
Da) ; (3) One missed cleavage was allowed; (4) Peptide ion tolerance: 15 ppm; (5) Fragment ion

tolerance: $25 \mathrm{ppm}$. Identified $\mathrm{Y} 1$ peptides from the first LC-MS run and the raw data from the second LC-MS run were imported to pGlyco for glycopeptide matching and scoring. All identified glycopeptides were manually checked by GlycoWorkbench Software developed by the EUROCarbDB[24]. The nomenclature of glycans is used according to Essentials of Glycobiology[25] and the abbreviations are used according to the NIBRT GlycoBase.

\section{2 \\ 3 Results and Discussion}

Both types of fucosylation structures of N-linked glycoproteins, core- and antennary-fucosylation, have been considered indicative in various cancers as biomarkers[1]. It is often difficult to distinguish the two structures. We thus sought to develop a method to distinguish core- and antennaryfucosylation at each glycosite of the target protein. A work-flow of this study is shown in Figure 1. Briefly, standard serum protein alph-1-antitrypsin (A1AT) was digested into peptides which were then treated by sialidase/galactosidase double digestion for glycan truncation. The truncated glycopeptides were semi-enriched and desalted by a $3 \mathrm{~K}$ membrane and analyzed by direct LC-MS/MS. The coreand antennary-fucosylation of A1AT was thus successfully distinguished and quantified.

The overall fucosylation level of serum proteins is quite low[26]. With the routine mass spectrometry settings for peptide analysis, in-source collision-induced dissociation (also called nozzle-skimmer dissociation, abbreviated as in-source dissociation herein) of glycopeptides would occur. This is a process where an ion dissociates as a result of collisional excitation during ion transfer from an atmospheric pressure ion source to the vacuum chamber of the mass spectrometer[27]. In our experiment in-source dissociation of glycopeptides was found to severely affect the identification and semi-quantification of the low abundance fucosylated peptides. However, there is so far no detailed analysis of this problem. Taking the most abundant glycan modification type A2 on one glycopeptide of A1AT for example, A2 should have 3 of Hex (mannose), 4 of HexNAc (GlcNAc), 0 
of NeuAc, 0 of NeuGlc and 0 of dHex after sialidase/galactosidase double digestion, abbreviated as 34000 herein. As shown in Figure 2, more than 20\% of 34000 glycans were decayed into 23000 (calculated as $\mathrm{XIC}_{23000} / \mathrm{XIC}_{34000}$ ) with the "universal" method settings for peptide analysis on Thermo Scientific Orbitrap Fusion mass spectrometers developed by Thermo Scientific (ion transfer tube temperature $=300^{\circ} \mathrm{C}, \mathrm{RF}=30 \%$ ) [28] or harsher settings. A series of MS settings for glycopeptides analysis were thus optimized. We found that with lower temperature and lower $R F\left(150{ }^{\circ} \mathrm{C}, 20 \%\right)$, the in-source dissociation of glycopeptides was reduced to less than $3 \%$, whereas the signal of corefucosylated peptide with 34001 glycan did not reduce significantly. We also found that higher spray voltage (spray voltage $>2300 \mathrm{~V}$ ) provided better signal but also increased in-source dissociation. Therefore the lowest spray voltage $2300 \mathrm{~V}$ for a stable spray was used. This optimized setting was used further to identify site-specific glycosylation and to semi-quantify core- and antennaryfucosylation of A1AT glycopeptides.

The direct LC-MS/MS strategy is shown in Figure 3, where first glycopeptides were selected by the detection of oxonium ion 138.05 with low energy HCD MS2 (Figure 3A); then the Y1 ion from the glycopeptide fragment was subjected to high energy HCD MS3 (Figure 3B) for peptide sequencing; while the selected glycopeptide was subjected to CID MS2 (Figure 3C) for glycan structure analysis; and the entire procedure is summarized in Figure 3D. The HCD collision energy (CE) of the first step was found to be crucial for fragmentation of glycopeptides. It was optimized for improved detection of oxonium ions and improved selection of the Y1 ion. As shown in Figure 4, either non-fucosylated or core-fucosylated or antennary-fucosylated biantennary glycopeptides with Asn271, low energy HCD MS2 with CE24 (among series of HCD from CE20 to CE32) provided the strongest Y1 ion fragment. This optimal CE seems to be irrelevant with glycan structures or peptide sequences. As shown for glycopeptides (with Asn271), either non-fucosylated or core-fucosylated or antennaryfucosylated structures have the same optimal CE (Figure 4), where the other 2 glycopeptides (with Asn107 or Asn70) of A1AT also have the same optimal CE (supporting information Figure S1). 
In contrast, the HCD CE for peptide annotation of the second step and the CID CE for glycan annotation of the third step were found not to be that sensitive. HCD MS3 with either CE35, 38 or 40 showed similar fragmentation patterns (shown in Figure 5), where HCD MS3 CE35 resulted in a somewhat stronger signal for higher $\mathrm{m} / \mathrm{z}$ fragments. Both CID CE30 and CE35 provided similar fragmentation of the glycan structures of glycopeptides (Figure 6). Thus in the following experiment, low energy HCD CE24, CID CE30 and high energy HCD CE 35 were used respectively.

Sialidase releases $\alpha 2-3,6,8,9 \mathrm{~N}$-acetylneuraminic acid leaving galactose as the terminal of the Nglycan. Subsequently, $\beta$-galactosidase cleaves $\beta 1-3,4$ galactose on condition that no fucose is bound to the subterminal $\mathrm{N}$-acetylglucosamine in an $\mathrm{N}$-glycan, thus providing a means to distinguish corefucosylation and antennary fucosylation[19]. The core-fucosylated and the antennary-fucosylated glycopeptides have the same $m / z$ in a sialidase digested sample (Figure 7.B), thus the spectrum of the sialidase digested sample is a mixture of core- and antennary-fucosylated peptides (Figure 7.B1). By contrast, core-and antennary-fucosylated peptides in the sialidase/galactosidase double digested sample have different $\mathrm{m} / \mathrm{z}$ (Figure 7.A). Therefore with sialidase/galactosidase double digestion on glycopeptides the two types of fucosylation are distinguished without further MS/MS analysis or extensive sequential exoglycosidase digestion, similar to previous work at the glycan level[19]. Also, with sialidase/galactosidase digestion, the retention time of an antennary-fucosylated glycopeptide is earlier than its corresponding core-fucosylated glycopeptides (Figure 7.A), indicating that the addition of a galactose enhanced its hydrophilicity. We found that not only the elution time but also the fragmentation patterns of core- and antennary-fucosylated glycopeptides were different. In the CID MS/MS spectrum of core-fucosylated glycopeptides, several core-fucosylated glycopeptide fragments were observed in a cluster (Figure 7.A1); whereas in the CID MS/MS spectrum of an antennary-fucosylated glycopeptide, pep-43001, pep-43000 and pep-33001 always appear as the three strongest fragments (Figure 7.A2). This difference of fragmentation patterns of core- and antennaryfucosylation was found in other glycopeptides as well (Figure S2). Therefore, using sialidase/galactosidase double digestion, a solid differentiation can be made between core- and 
antennary-fucosylated peptides using direct LC-MS/MS analysis. Traditional exoglycosidase with fucosidase $\alpha 1-2,3,4,6$ and fucosidase $\alpha 1-3,4$ were further applied on the sialidase/galactosidase double digested glycopeptides, showing the efficacy of this strategy (Figure S3).

In our previous study, we analyzed the glycans cleaved from the glycoprotein A1AT and found that A1AT has 12 different glycan structures after sialidase/galactosidase double digestion[19]. In this study, we identified 10 of these structures on specific glycosylation sites. Their retention times on a C18 column and their relative intensities are shown in Table 1. Low energy HCD MS2, high energy MS3 and CID MS2 spectra of all identified glycopeptides are shown in supporting information Figure S2. Glycopeptide1 ADTHDEILEGLNFnLTEIPEAQIHEGFQELLR with Asn107 (modified amino acid is shown in lower ease) has the most various glycan modification types including A2, FA2, A2FG, A3, FA3, A3FG, A4, FA4, A4FG, A3F2G2, whereas the other two sites have fewer glycan modification types (glycopeptide2 YLGnATAIFFLPDEGK with Asn271: A2, FA2, A2FG, A3, A3FG and glycopeptide3 QLAHQSnSTNIFFSPVSIATAFAMLSLGTK with Asn70: A2). The extent to which each site is glycosylated may possibly depend on the protein structure or proximity of the site to certain amino acids or to the N/C terminus[22]. As shown in Figure 8 (crystal structure from [29]), all three sites are located at the protein surface and in loops, among which Asn107 is almost in the center of a big loop and may be more accessible by various glycosyltransferases while Asn271 and Asn70 are closer to the alpha helix or beta sheet structures and have smaller spaces. This may partially explain why Asn107 has the most various glycosylation modification.

As expected, after sialidase/galactosidase double digestion, the extra fucose of core-fucosylated glycopeptides made the glycopeptide more hydrophilic, thus its elution from the C18 column was earlier than its corresponding non-fucosylated glycopeptides for bi- and tri-antennary glycan modifications. An extra galactose made the antennary-fucosylated bi-antennary glycopeptide even more hydrophilic compared to its corresponding core-fucosylated case. However, a further galactose and/or fucose did not make the tri- or tetra- antennary glycopeptides significantly more hydrophilic and the elution times of all fucosylated tri-antennary glycopeptides or all tetra-antennary 
glycopeptides were similar on a very slow elution gradient $(0.2 \% \mathrm{ACN} / \mathrm{min})$. It can be concluded that after sialidase/galactosidase double digestion, the hydrophobicity of glycopeptides is mainly determined by its peptide backbone and only a slight hydrophobicity change was found in biantennary glycans. This change may be due to the fact that bi-antennary glycans have fewer sugar units and one or two extra sugars may contribute overall more hydrophobicity to the glycopeptides compared to tri- and tetra- antennary glycans.

Our previous study of glycans showed that the nonfucosylated bi- and tri-antennary glycans are the top two most abundant glycan structures of A1AT, comprising $35.8 \%$ and $25.2 \%$ respectively[19]. In this study of glycopeptide1 and glycopeptide2, we found the bi- and tri-antennary glycan modification on Asn 107 comprised $48 \%$ and $34 \%$ respectively whereas those on Asn271 comprised $97 \%$ and $1 \%$ respectively(Table 1). The analysis of glycopeptide3 showed that there was only A2 glycan modification on Asn70; thus we consider the glycan modification on Asn70 does not contribute much to the overall glycosylation of A1AT protein. A chi-square test is used for comparison of the relative peak intensity of major glycan modification types between glycan types cleaved from A1AT protein (data from previous result[19]) and glycan modification types of glycopeptide1 (with Asn107) or glycan modification types of glycopeptide2 (with Asn271) (Table 2). The glycan types cleaved from A1AT protein are significantly different from the glycan modification types on Asn271 ( $p$ value $<0.01$ ), but not different from those on Asn107 ( $p$ value=0.97), indicating that the glycosylation level of Asn107 overwhelms the other 2 sites and contributes more to the glycan structure of the A1AT protein The core- and antennary-fucosylation level of the two sites also varied significantly, where the antennary-fucosylated tri-antennary glycan was the most abundant fucosylation type on Asn107 but it was negligible on Asn271.

One study with classical lectin blot assay found that the up-regulation of core-fucosylated but not antennary-fucosylated A1AT could be indicative for hepatocellular cancer diagnosis[20] while antennary-fucosylation of A1AT indicates inflammation especially in $\mathrm{HBV}$-infected patients[22]. Our previous glycan study has indicated that bi-antennary core-fucosylation was the most abundant core- 
fucosylation type of A1AT protein. From Table 1 we may deduce that if there were alteration in A1AT core-fucosylation types in patients, the bi-antennary core-fucosylation on Asn107 is most likely the possible target that can be precisely monitored and quantified by mass spectrometry. Another classical lectin blot-based study showed that the overall increase of A1AT fucosylation level was able to distinguish lung adenocarcinoma from benign diseases or other lung cancer subtypes[21]. The strategy developed in this study would enable the identification and quantification of core- and antennary-fucosylation on specific sites of A1AT. In future work, this methodology will be used to study changes in serum A1AT glycosylation during the progression of various cancers. The more precise fucosylation analysis with site-specific information should provide improved diagnostic value. Also, this strategycan be applied to the study of other key glycoproteins during the progression of various diseases.

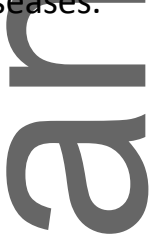

4 Concluding Remarks

We have developed a pipeline to study the glycosylation of A1AT to identify the presence of coreversus antennary-fucosylation without separating glycans and peptides from glycopeptides. This was performed on a standard protein A1AT which was digested by trypsin followed by sialidase/galactosidase digestion. Galactosidase removes terminal galactose residues in an N-glycan except when the subterminal $\mathrm{N}$-acetylglucosamine is modified by fucosylation, thus providing a means to distinguish core-fucosylation and antennary-fucosylation. The sites and structures of glycans could be determined simultaneously by this procedure. In total, we identified 1 glycan structure (A2) on Asn70 of glycopeptide QLAHQSnSTNIFFSPVSIATA, 10 glycan structures (A2, FA2, A2FG, A3, FA3, A3FG, A4, FA4, A4FG and A3F2G2) on Asn107 of glycopeptide ADTHDEILEGLNFNLTEIPEAQIHEGFQE

LLR, and 5 glycan structures (A2, FA2, A2FG, A3 and A3FG) on Asn271 of glycopeptide YLGnATAIFFLPDEGK. We believe that this methodology will be widely used to identify and quantify 
core- and antennary-fucosylation on A1AT or other key glycoproteins during the progression of various diseases.

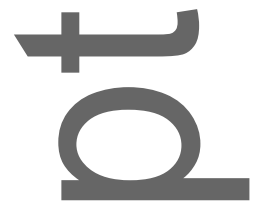

Acknowledgements: This work was funded by the National Natural Science Foundation of China (81601828). Thanks for the software support from Dr. Zeng WF from the pGlyco group in the Institute of Computing Technology, CAS, Beijing. Also we thank the support of the University Research Facility in Chemical and Environmental Analysis and the University Research Facility in Life Sciences of Hong Kong Polytechnic University. Partial support was also provided by the National Cancer Institute under grant no. R01 CA160254 (D.M.L.)

There are no conflicts of interest to declare.

\section{Supporting Information Available}

Figure S1.Either glycopeptide1, glycopeptide2 or glycopeptide3 have the same optimal low energy HCD CE2

Figure S2. Low energy HCD MS2, high energy HCD MS3 and CID MS2 spectra of all identified glycopeptides with various glycan modification types: glycopeptide1 (with Asn107), glycopeptide2 (with Asn271) and glycopeptide3 (with Asn70).

Figure S3. XIC (Extracting Ion Current) of fucosylated glycopeptides in sialidase/galactosidase digested A1AT sample, in sialidase/galactosidase/fucosidase $\alpha 1-2,3,4,6$ digested A1AT sample and in sialidase/galactosidase /fucosidase $\alpha 1-3,4$ digested A1AT sample. 
Table 1. Summary of glycopeptides of A1AT with truncated glycan and \% relative peak area of coreand antennary-fucosylation of each site.

\begin{tabular}{|c|c|c|c|c|c|}
\hline Glycopeptide Experimental $\mathrm{m} / \mathrm{z}$ & $\begin{array}{l}\text { Charge } \\
(z)\end{array}$ & $\begin{array}{l}\text { Theoretical } \\
\mathrm{m} / \mathbf{z}\end{array}$ & $\begin{array}{l}\text { delta } \\
\text { ppm }\end{array}$ & $\begin{array}{l}\text { Retention } \\
\text { Time(min) }\end{array}$ & $\begin{array}{l}\% \\
\text { Relative } \\
\text { peak area }\end{array}$ \\
\hline \multicolumn{6}{|c|}{$\begin{array}{l}\text { GLYCOPEP1 ADTHDEILEGLNFnLTEIPEAQIHEGFQELLR } \\
\text { with Asn107 }\end{array}$} \\
\hline A2 1248.8282 & $4+$ & 1248.8334 & 4.2 & 49.59 & 48.4 \\
\hline \begin{tabular}{|l|l}
1285.3411 \\
\end{tabular} & $4+$ & 1285.3479 & 5.3 & 49.51 & 2.1 \\
\hline 1325.8546 & $4+$ & 1325.8611 & 4.9 & 49.37 & 1.1 \\
\hline 1299.5972 & $4+$ & 1299.6033 & 4.7 & 49.51 & 33.8 \\
\hline 1069.0868 & $5+$ & 1069.0957 & 8.4 & 49.37 & 0.3 \\
\hline 1376.6236 & $4+$ & 1376.6309 & 5.3 & 49.37 & 11.3 \\
\hline 1453.6519 & $4+$ & 1453.6586 & 4.6 & 49.37 & 0.3 \\
\hline 1350.3662 & $4+$ & 1350.3731 & 5.1 & 49.37 & 2.3 \\
\hline 1386.8769 & $4+$ & 1386.8876 & 7.7 & 49.37 & 0.2 \\
\hline 1142.1147 & $5+$ & 1142.1222 & 6.5 & 49.37 & 0.2 \\
\hline \multicolumn{6}{|l|}{$\begin{array}{l}\text { GLYCOPEP2 } \\
\text { with Asn271 YLGnATAIFFLPDEGK }\end{array}$} \\
\hline A2 1019.4596 & $3+$ & 1019.4657 & 6.0 & 32.21 & 97.2 \\
\hline \begin{tabular}{l|l|} 
& 1068.1455 \\
\end{tabular} & $3+$ & 1068.1517 & 5.8 & 31.83 & 0.2 \\
\hline 1122.1628 & $3+$ & 1122.1693 & 5.8 & 31.69 & 1.4 \\
\hline 1087.1553 & $3+$ & 1087.1589 & 3.3 & 31.86 & 1.0 \\
\hline \begin{tabular}{l|l} 
A3FG & 1189.8561 \\
\cline { 1 - 2 }
\end{tabular} & $3+$ & 1189.8624 & 5.3 & 31.36 & 0.2 \\
\hline \multicolumn{6}{|c|}{$\begin{array}{l}\text { GLYCOPEP3 } \\
\text { with Asn70 }\end{array}$} \\
\hline \begin{tabular}{l|l|l} 
A2 & 1125.2794
\end{tabular} & $4+$ & 1125.2850 & 4.9 & 53.6 & null \\
\hline
\end{tabular}

Table 2. A chi-square test is used for comparison of the relative peak intensity of major glycan modification types between glycan types cleaved from A1AT protein (data from previous result[19]) and glycan modification types on glycopeptide1 (with Asn107) or glycopeptide2 (with Asn271).

\begin{tabular}{|c|c|c|c|c|c|c|}
\hline & \multicolumn{5}{|c|}{$\%$ Relative peak area } & \multirow{2}{*}{$\begin{array}{l}\text { chi-square } \\
\text { test } p \text { value }\end{array}$} \\
\hline & A2 & FA2 & $\mathrm{A} 2 \mathrm{~F}$ & A3 & A3FG & \\
\hline GLYCAN & 35.8 & 1.8 & 0.5 & 25.2 & 11.3 & - \\
\hline $\begin{array}{l}\text { GLYCOPEP1 } \\
\text { with Asn107 }\end{array}$ & 48.4 & 2.1 & 1.1 & 33.8 & 11.3 & 0.97 \\
\hline $\begin{array}{l}\text { GLYCOPEP2 } \\
\text { with Asn271 }\end{array}$ & 97.2 & 0.2 & 1.4 & 1 & 0.2 & $<0.01$ \\
\hline
\end{tabular}


${ }^{*} p$ value $<0.05$ is considered significant

\section{Figure Captions}

Figure 1. Work-flow of the experiment for determining glycosylation of A1AT. The glycosylated A1AT was first digested into peptides, followed by glycan truncation by sialidase/galactosidase double digestion. Glycopeptides were subjected to direct LC-MS/MS analysis without cleaving glycans.

Figure 2. In-source dissociation of A1AT glycopeptides (Asn271) with A2 and FA2 glycan modification types under various settings of ion transfer tube temperature, spray voltage and RF\%.

Figure 3. Spectra of A1AT glycopeptide (Asn271) with A2FG glycan modification type: (A) low energy HCD MS2 spectrum; (B) low energy HCD triggered high energy HCD MS3 spectrum; (C) low energy HCD triggered CID MS2; (D) Illustration of the fragmentation of the glycopeptide.

Figure 4. MS2 fragmentation patterns of glycopeptides (Asn 271) with A2, FA2 and A2FG glycan modification types under various low energy HCD collision energies, indicating that low energy HCD with CE24 provides the strongest Y1 ion (peptide+GlcNAc).

Figure 5. MS3 fragmentation patterns of Y1 ion (peptide+GlcNAc) of glycopeptides (Asn271) under various high energy HCD collision energies, indicating that high energy HCD with CE35 provides the best fragmentation profile.

Figure 6. MS2 fragmentation patterns of glycopeptides (Asn271) with A2, FA2 and A2FG glycan modification types under various CID collision energies, indicating that either CE30 or CE35 provides similar fragmentation profile of these glycopeptides.

Figure 7. Differentiation of core- and antennary-fucosylation of glycopeptides (Asn271) by sialidase/galactosidase digestion (A: retention time; A.1: spectrum of FA2; A.2: spectrum of A2FG). As a comparison, no differentiation was observed for sialidase-digested case (B: retention time; B.1 spectrum of $A 2 G 2(F))$. 
Figure 8. The three glycosylated sites Asn70, Asn107 and Asn271 of A1AT are labeled red and the detected peptides by mass spectrometry are labeled green in the 3D structure.

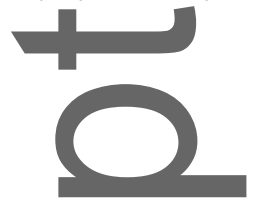

Figure 1.

1.

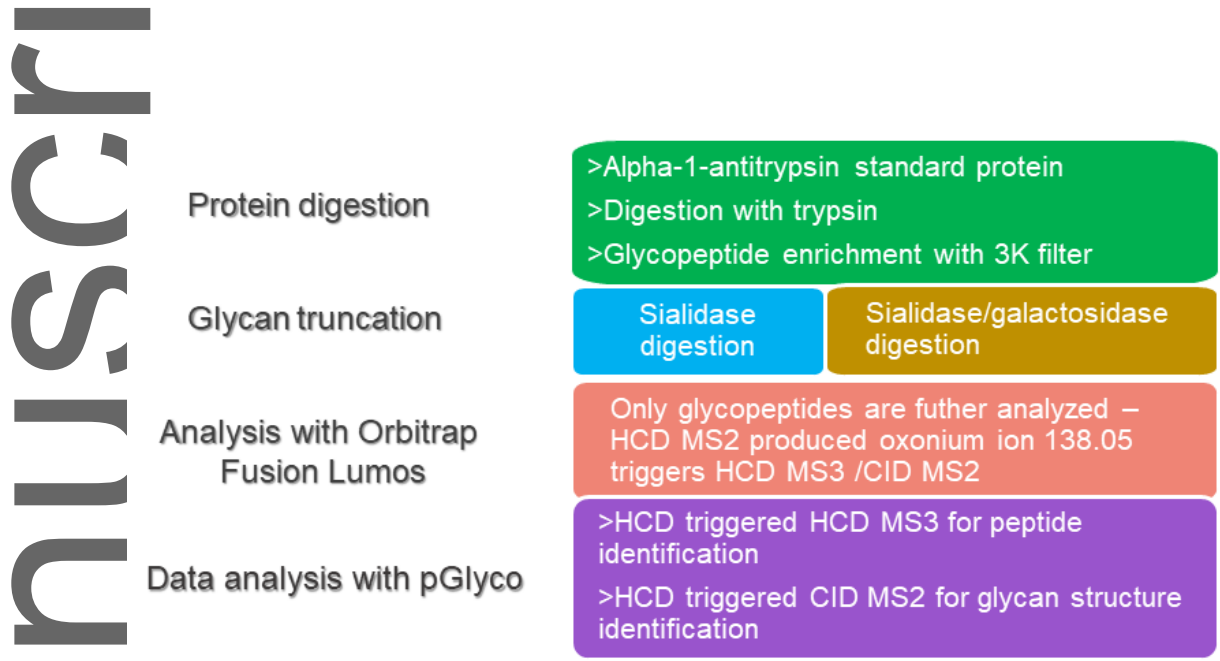

Figure 2.

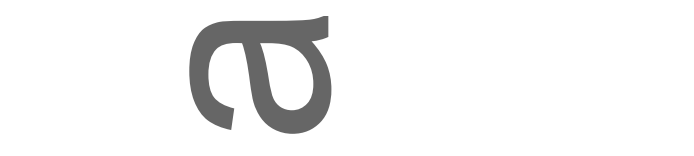

$>$ HCD triggered CID MS2 for glycan structure

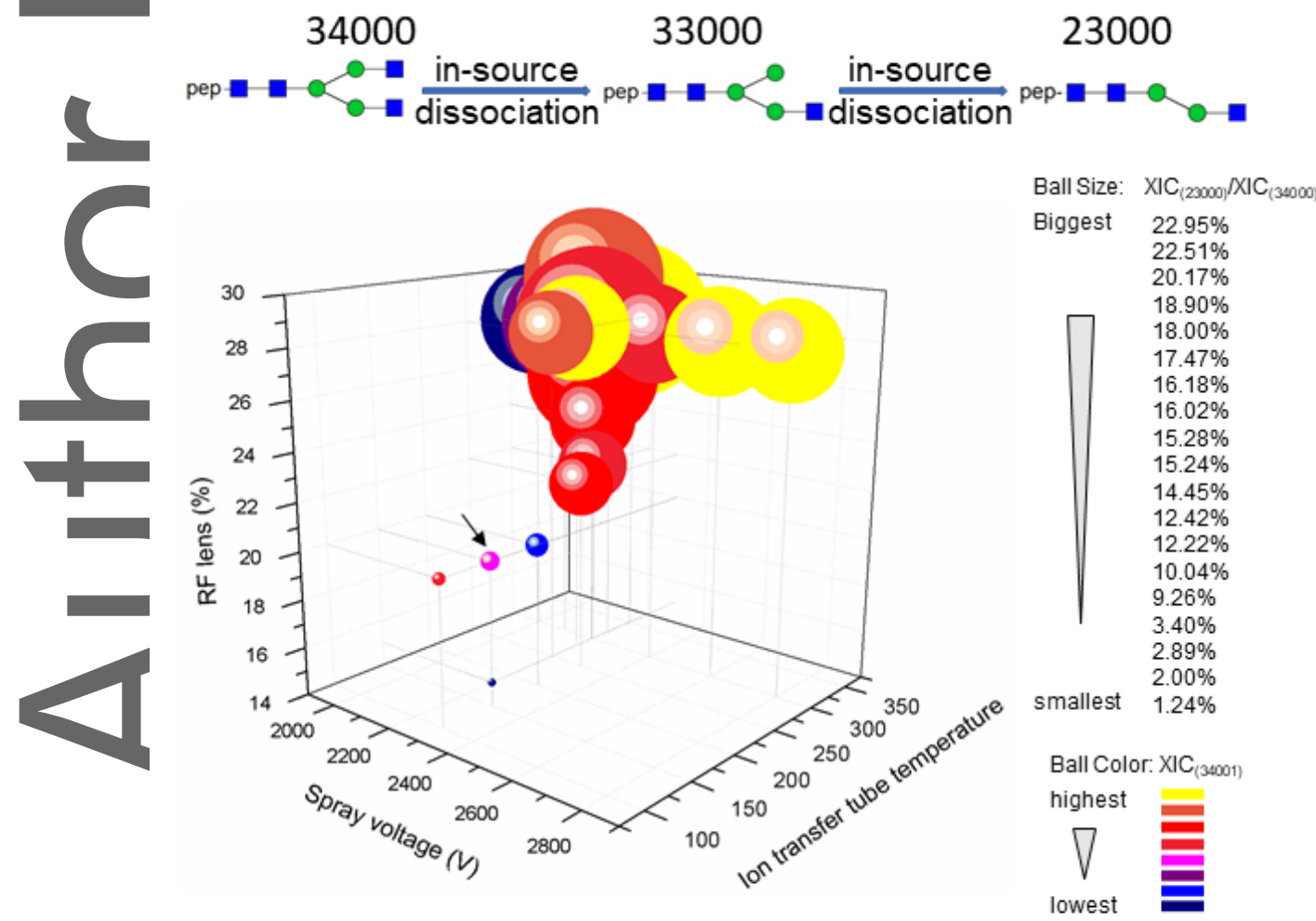

Relative low in-source decay and high intensity of fucosylated glycopeptide at spray voltage $2300 \mathrm{~V}$, ion transfer tube temperature $150^{\circ} \mathrm{C}$ and RF $20 \%$

This article is protected by copyright. All rights reserved 

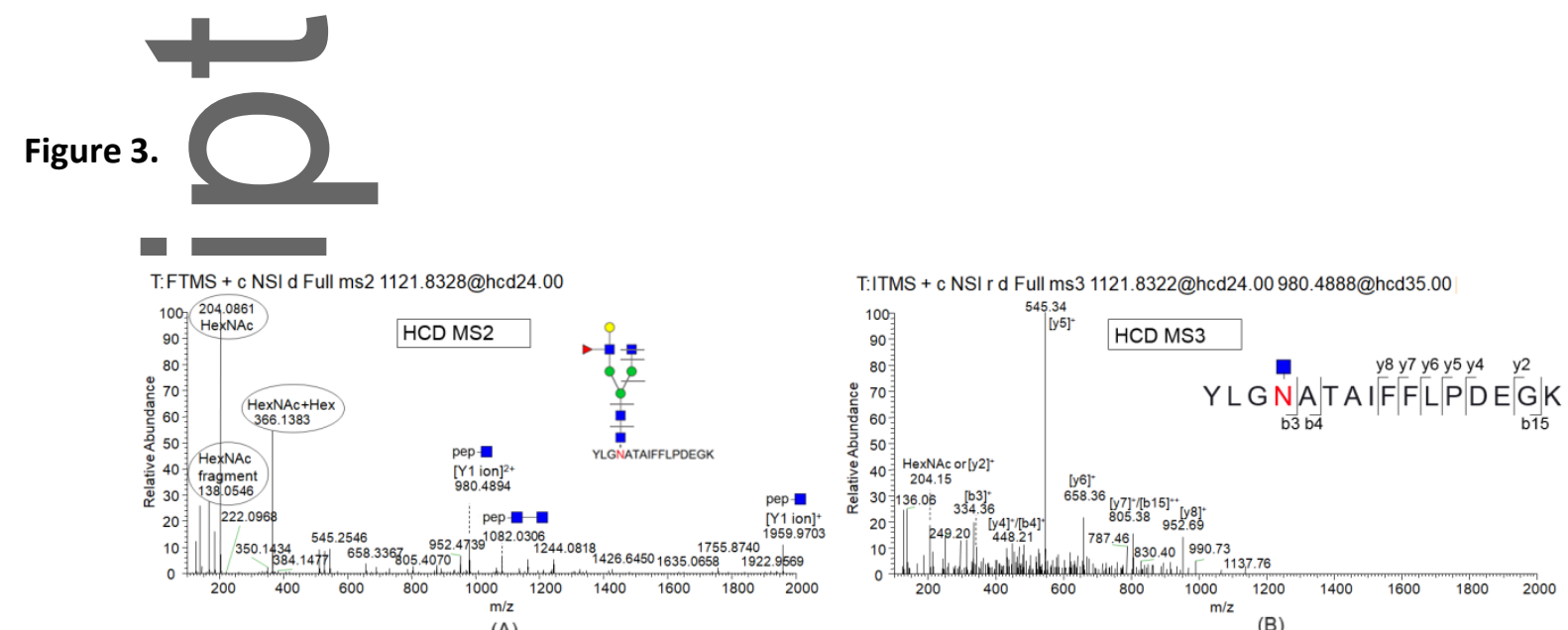

(A)

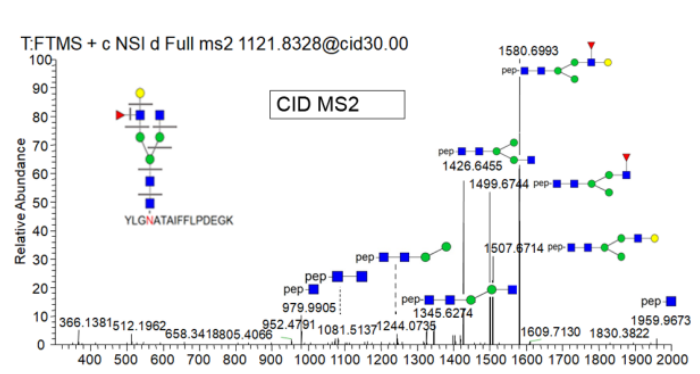

(C)

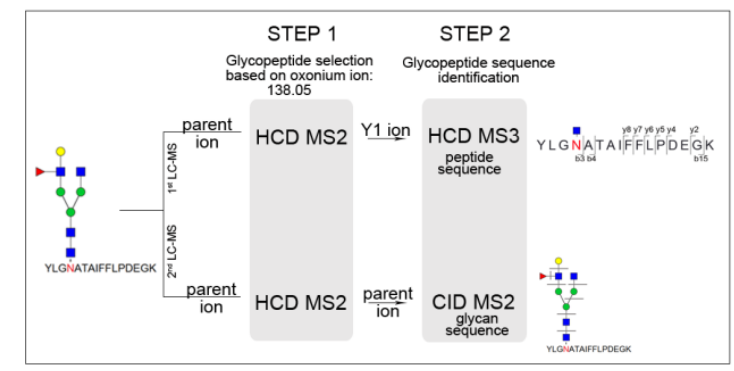

(D)
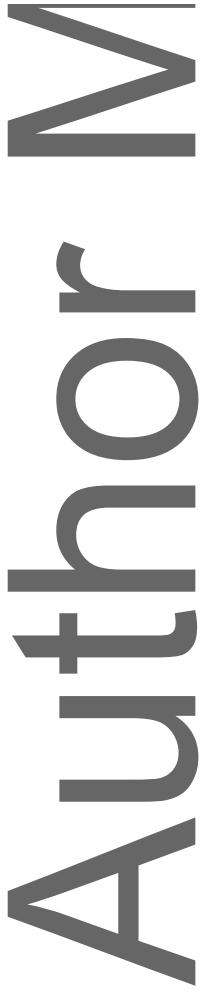
Figure 4.

(A) Glycopep-A2

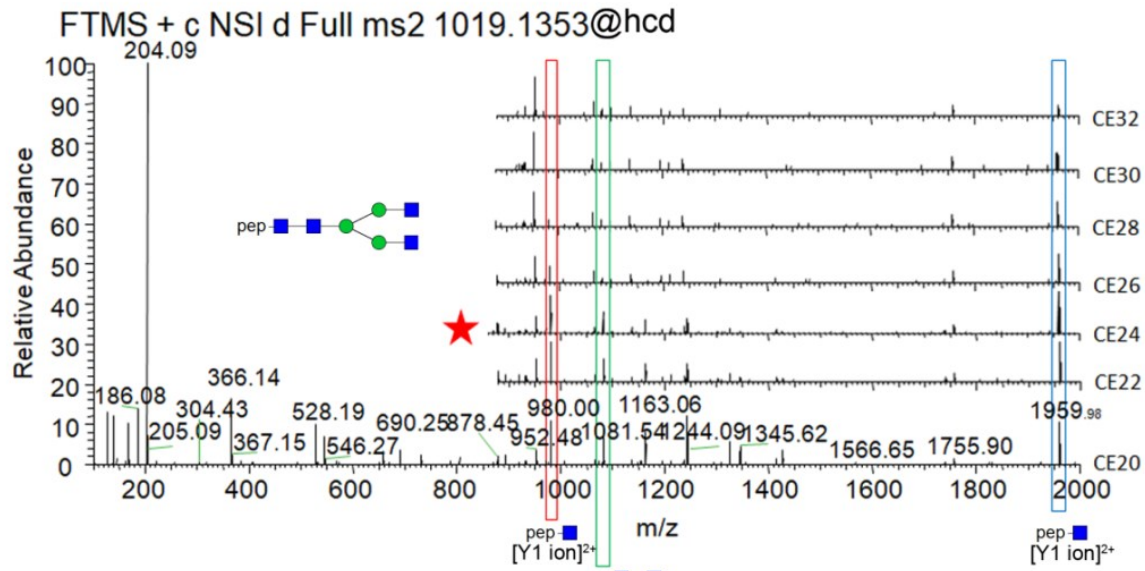

FTMS + c NSI d Full ms2 1068.1554@hcd

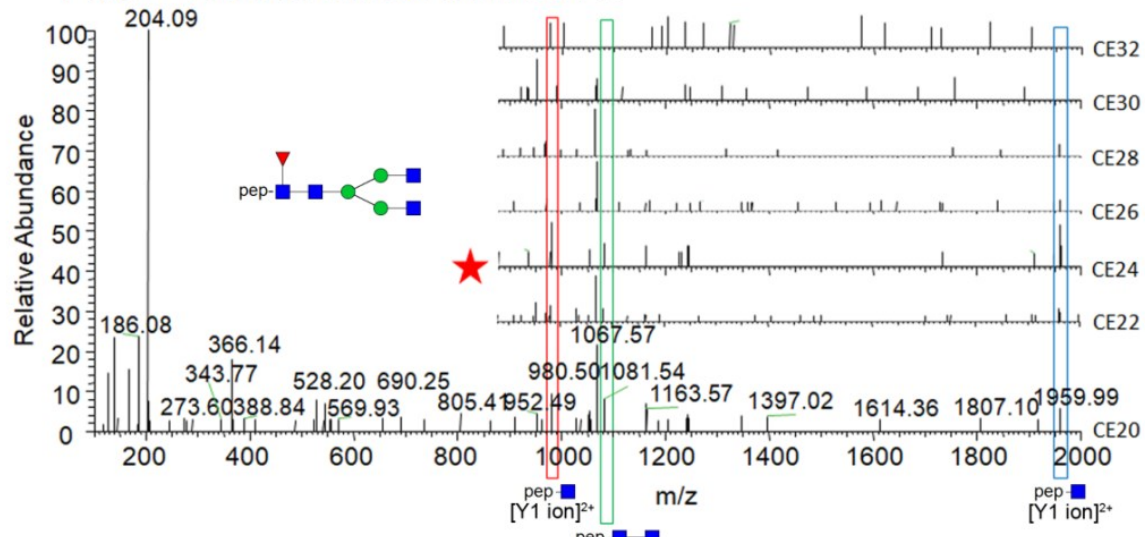

FTMS + c NSI d Full ms2 1121.7244@hcd

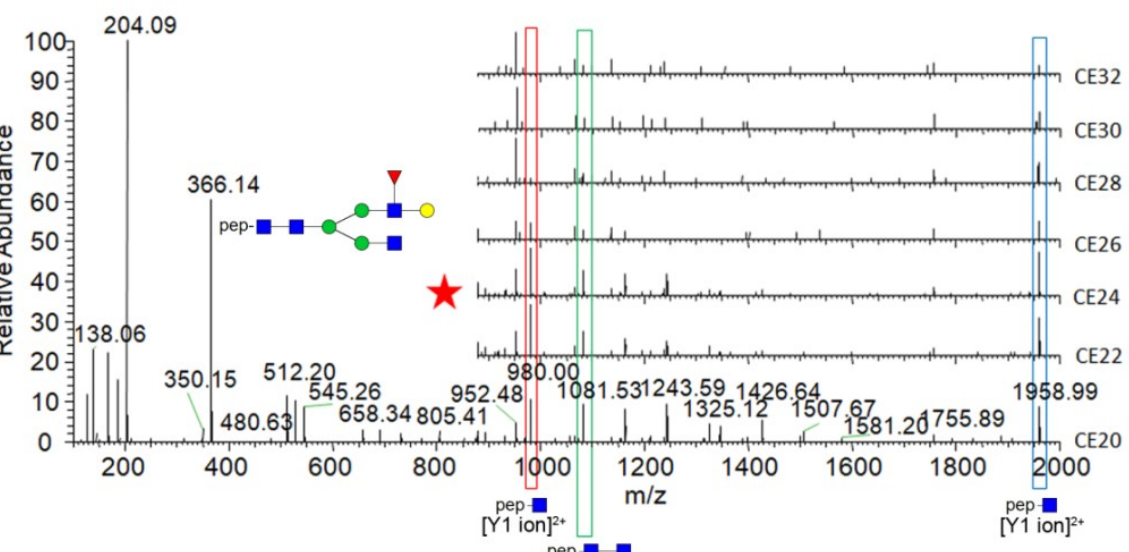

Figure 5.

This article is protected by copyright. All rights reserved 
ITMS + c NSI r d Full ms3 1019.1290@hcd24.00980.4941@hcd
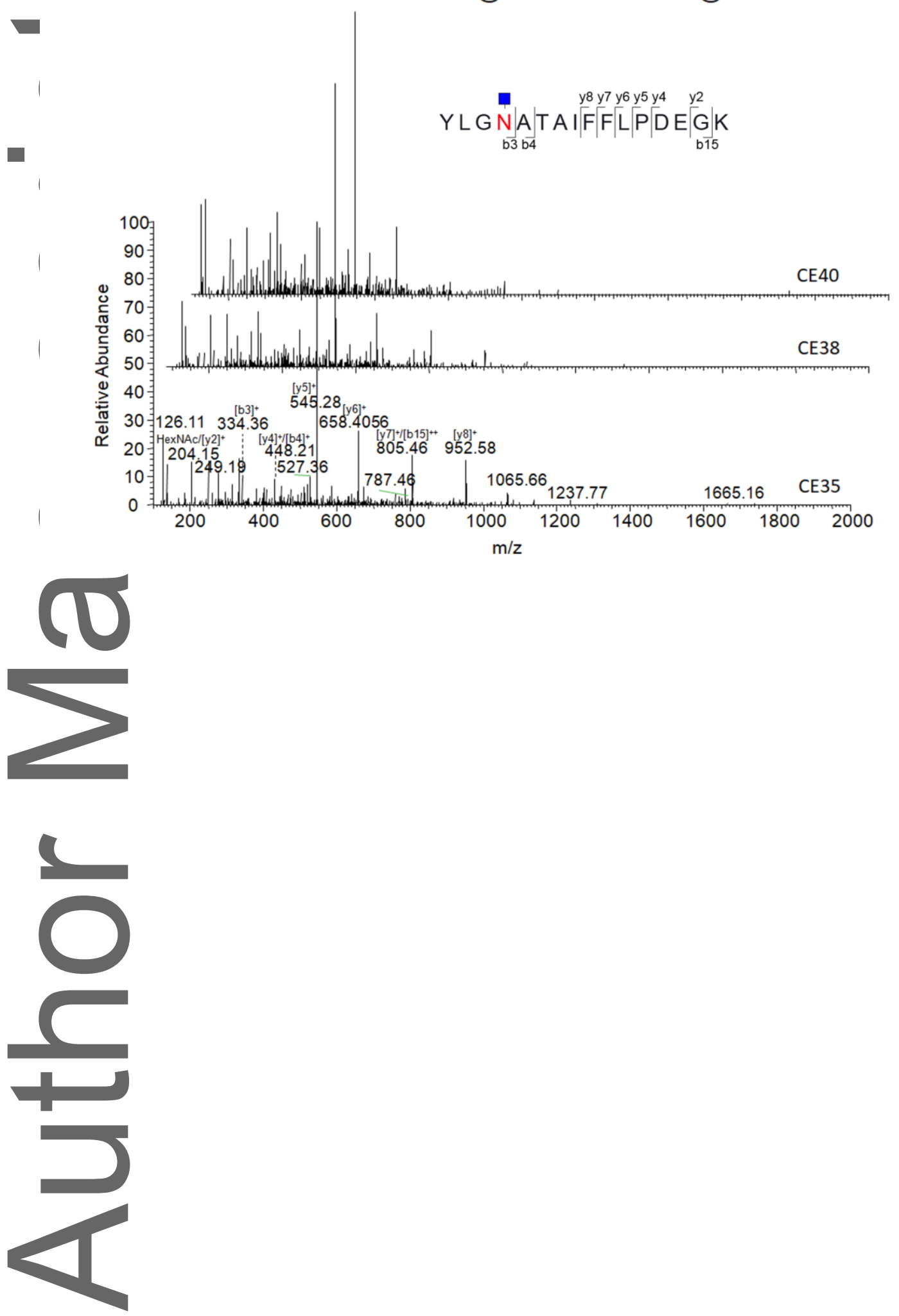
Figure 6.

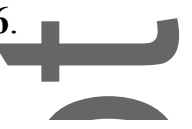

FTMS + c NSI d Full ms2 1019.1284@cid

(A) Glycopep-A2

(B) Glycopep-FA2

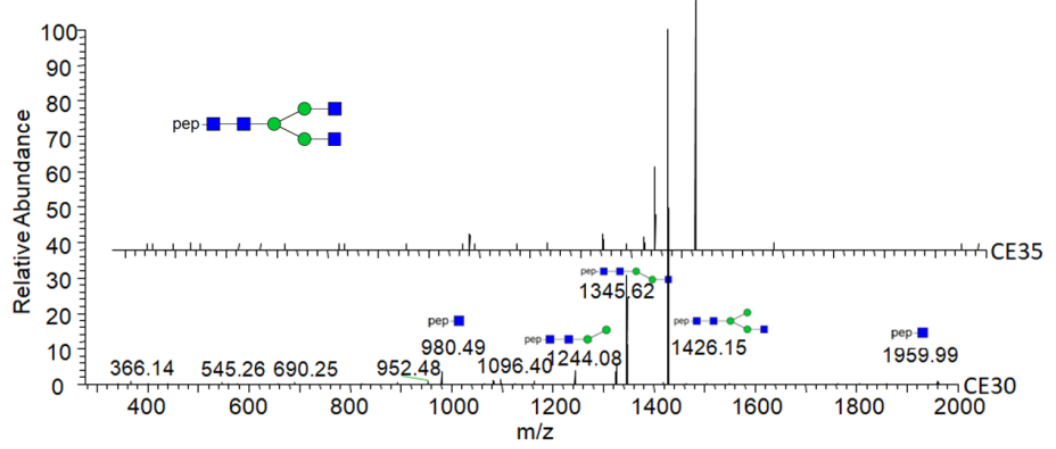

FTMS + c NSI d Full ms21067.8345@cid

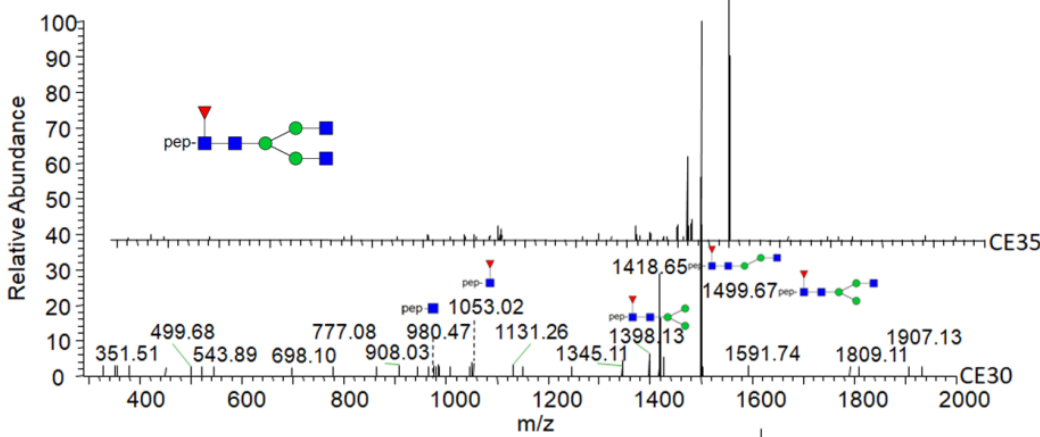

FTMS + c NSI d Full ms21121.8330@cid

(C) Glycopep-A2FG

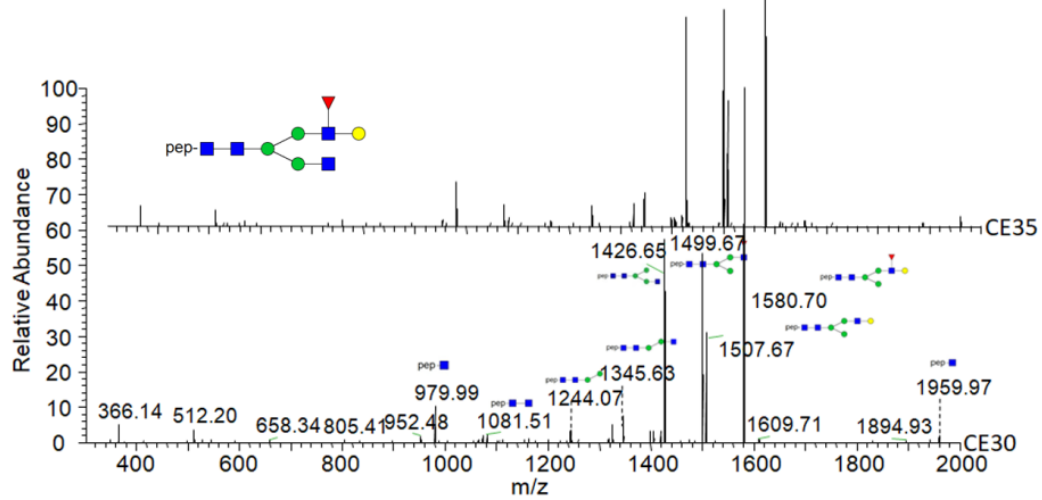




\section{Figure 7.}

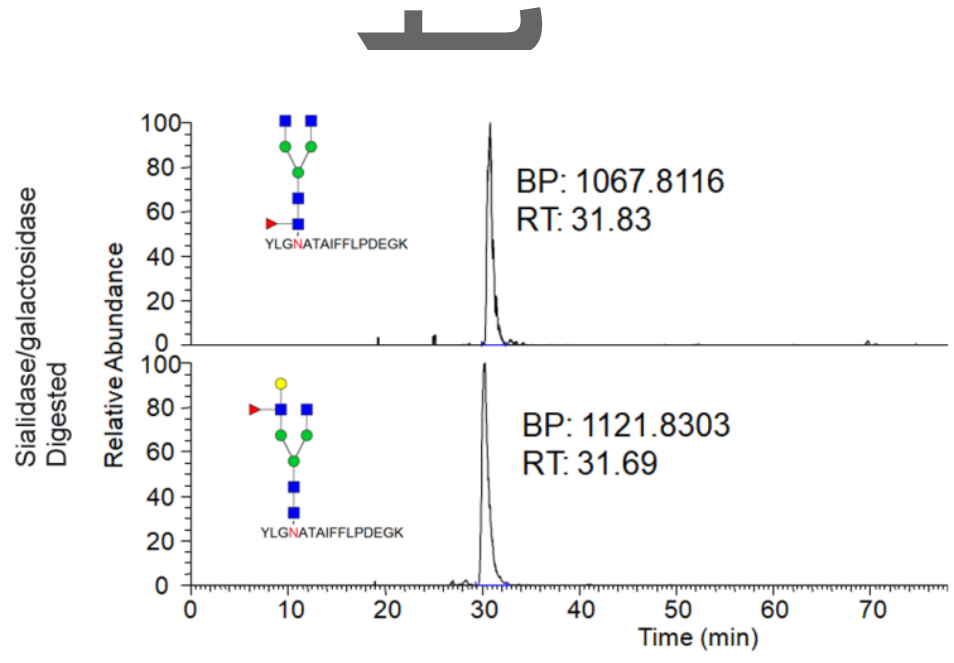

(A)

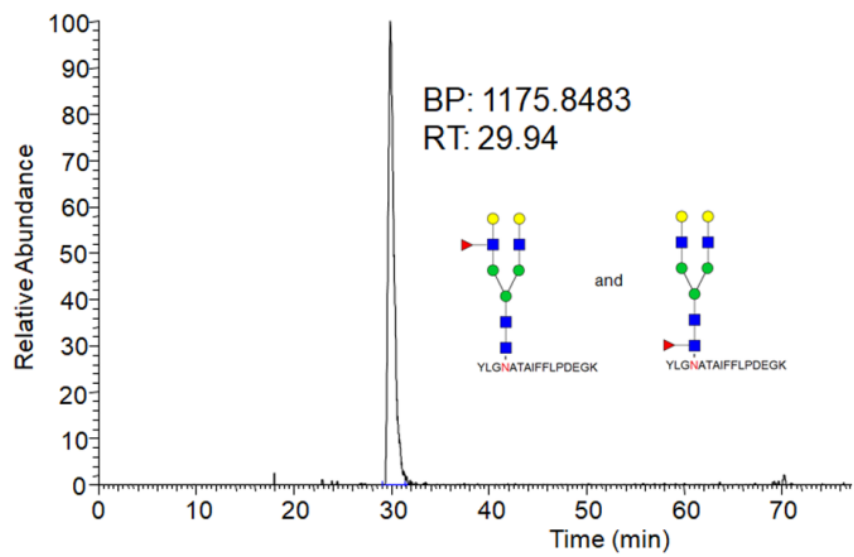

(B)

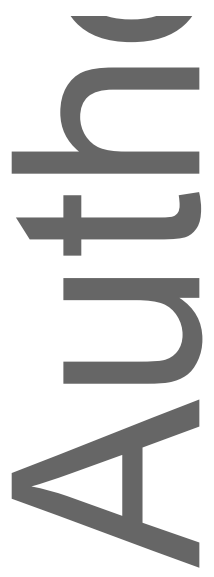

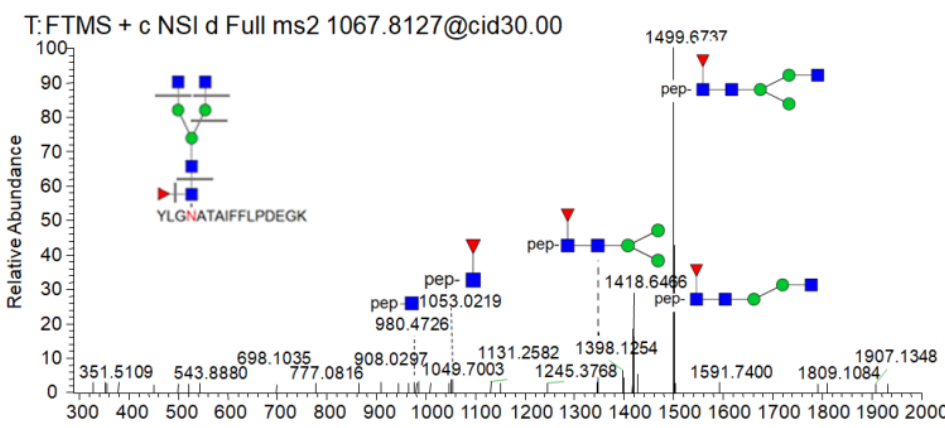
$\mathrm{m} / \mathrm{z} \quad$ (A.1)

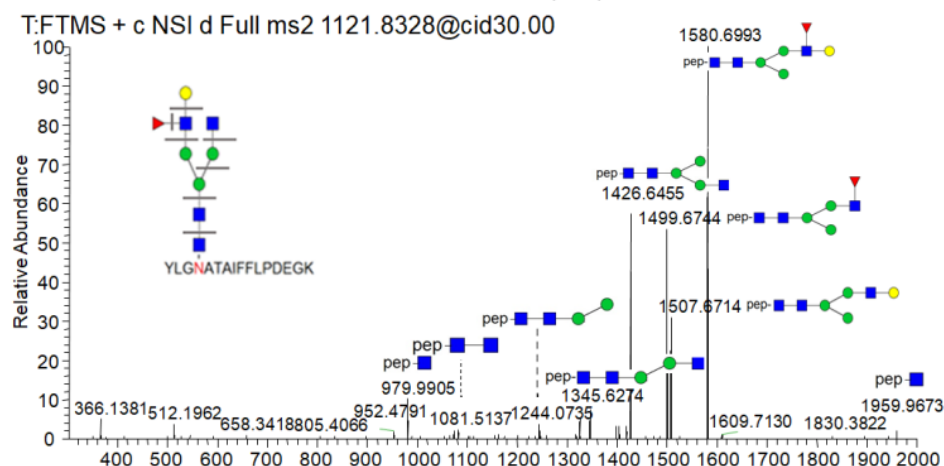
40050060070080090010001100120013001400150016001700180019002000 $\mathrm{m} / \mathrm{z} \quad$ (A.2)

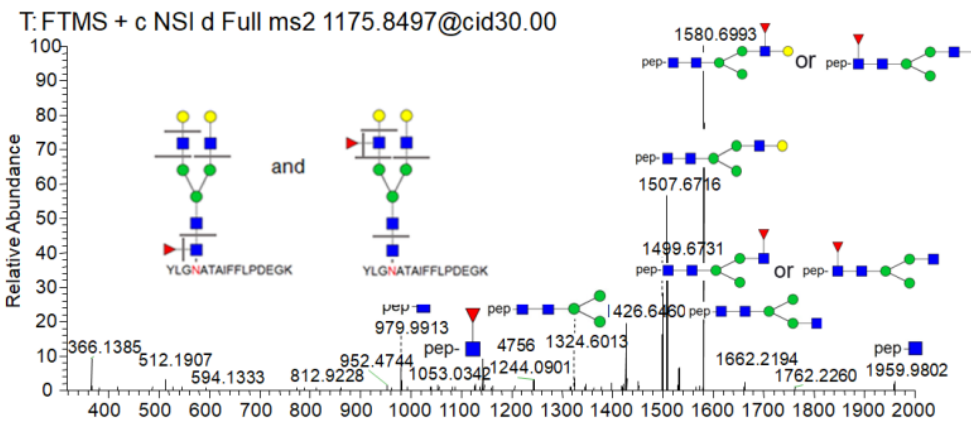
$\mathrm{m} / \mathrm{z} \quad$ (B.1) 
Figure 8.

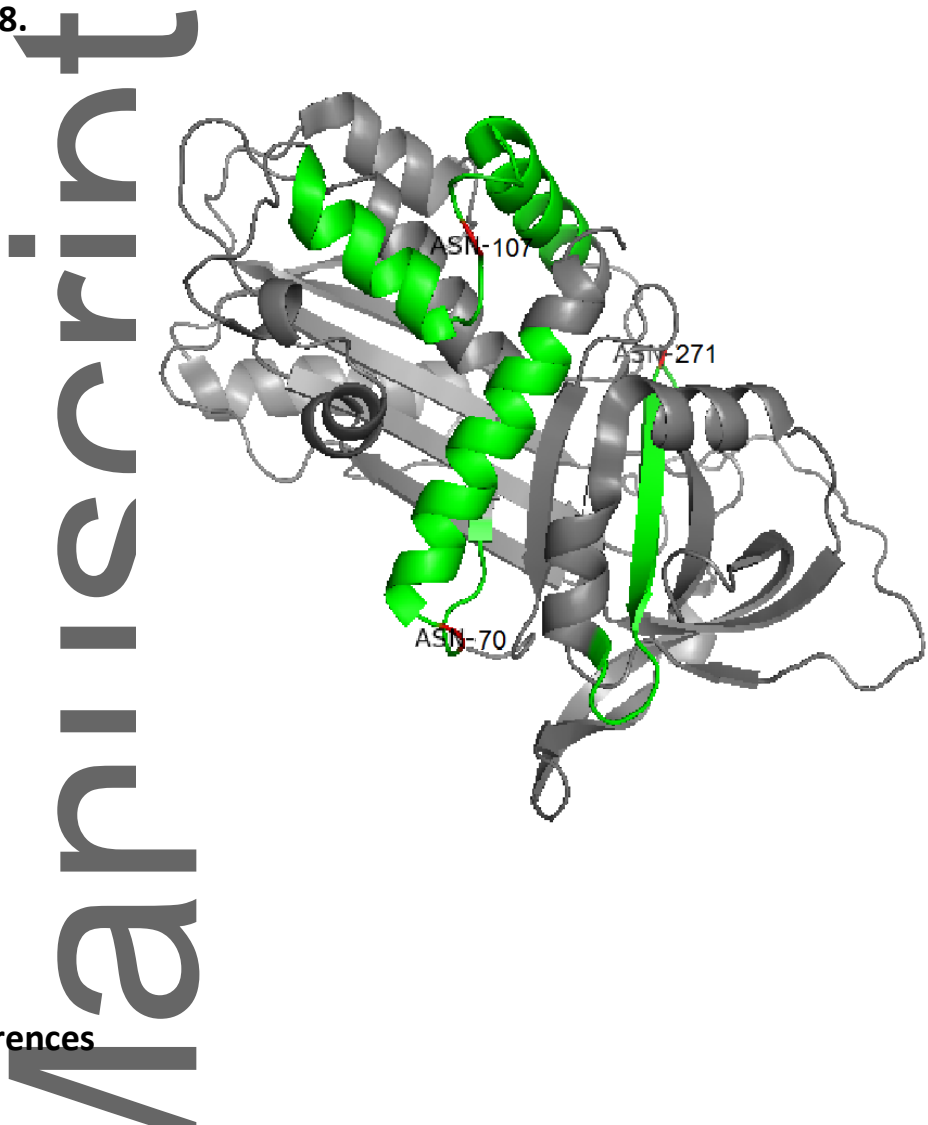

[1] Christiansen, M. N., Chik, J., Lee, L., Anugraham, M., Abrahams, J. L., Packer, N. H., Proteomics 2014, 14, 525-546.

[2] Pompach, P., Brnakova, Z., Sanda, M., Wu, J., Edwards, N., Goldman, R., Molecular \& cellular proteomics: MCP 2013, 12, 1281-1293.

[3] Marrero, J. A., Feng, Z. D., Wang, Y. H., Nguyen, M. H., Befeler, A. S., Roberts, L. R., Reddy, K. R., Harnois, D., Llovet, J. M., Normolle, D., Dalhgren, J., Chia, D., Lok, A. S., Wagner, P. D., Srivastava, S., Schwartz, M., Gastroenterology 2009, 137, 110-118.

[4] Goonetilleke, K. S., Siriwardena, A. K., European journal of surgical oncology : the journal of the European Society of Surgical Oncology and the British Association of Surgical Oncology 2007, 33, 266270.

[5] Guttman, A., Electrophoresis 1997, 18, 1136-1141.

[6] Szigeti, M., Bondar, J., Gjerde, D., Keresztessy, Z., Szekrenyes, A., Guttman, A., Journal of chromatography. B, Analytical technologies in the biomedical and life sciences 2016, 1032, 139-143.

[7] Desaire, H., Molecular \& cellular proteomics : MCP 2013, 12, 893-901.

[8] Chandler, K. B., Costello, C. E., Electrophoresis 2016, 37, 1407-1419.

[9] Wuhrer, M., Catalina, M. I., Deelder, A. M., Hokke, C. H., Journal of chromatography. B, Analytical technologies in the biomedical and life sciences 2007, 849, 115-128.

[10] Ma, C., Zhang, Q., Qu, J., Zhao, X., Li, X., Liu, Y., Wang, P. G., J Proteomics 2015, 114, 61-70.

[11] Yu, Q., Wang, B., Chen, Z., Urabe, G., Glover, M. S., Shi, X., Guo, L. W., Kent, K. C., Li, L., Journal of the American Society for Mass Spectrometry 2017. 
[12] Bern, M., Kil, Y. J., Becker, C., Current protocols in bioinformatics 2012, Chapter 13, Unit13 20. [13] Sun, S., Shah, P., Eshghi, S. T., Yang, W., Trikannad, N., Yang, S., Chen, L., Aiyetan, P., Hoti, N., Zhang, Z., Chan, D. W., Zhang, H., Nature biotechnology 2016, 34, 84-88.

[14] Liu, M. Q., Zeng, W. F., Fang, P., Cao, W. Q., Liu, C., Yan, G. Q., Zhang, Y., Peng, C., Wu, J. Q., Zhang, X. J. TU, H.J., Chi, H., Sun, R. X., Cao, Y., Dong, M. Q., Jiang, B. Y., Huang, J. M., Shen, H. L., Wong, C. C. L., He, S. M., Yang, P. Y., Nature communications 2017, 8, 438.

[15] Zeng, W. F., Liu, M. Q., Zhang, Y., Wu, J. Q., Fang, P., Peng, C., Nie, A., Yan, G., Cao, W., Liu, C., Chi, H., Sun, R. X., Wong, C. C., He, S. M., Yang, P., Scientific reports 2016, 6, 25102.

[16] Pompach, P., Ashline, D. J., Brnakova, Z., Benicky, J., Sanda, M., Goldman, R., J Proteome Res 2014, 13, 5561-5569.

[17] Wuhrer, M., Koeleman, C. A., Hokke, C. H., Deelder, A. M., Rapid communications in mass spectrometry: RCM 2006, 20, 1747-1754.

[18] Veillon, L., Huang, Y., Peng, W., Dong, X., Cho, B. G., Mechref, Y., Electrophoresis 2017, 38, 2100 2114.

[19] Yin, H., Zhu, J., Wu, J., Tan, Z., An, M., Zhou, S., Mechref, Y., Lubman, D. M., Electrophoresis 2016, 37, 2624-2632.

[20] Comunale, M. A., Rodemich-Betesh, L., Hafner, J., Wang, M., Norton, P., Di Bisceglie, A. M., Block, T., Mehta, A., PloS one 2010, 5, e12419.

[21] Liang, Y., Ma, T., Thakur, A., Yu, H., Gao, L., Shi, P., Li, X., Ren, H., Jia, L., Zhang, S., Li, Z., Chen, M., Glycobiology 2015,25, 331-340.

[22] McCarthy, C., Saldova, R., Wormald, M. R., Rudd, P. M., McElvaney, N. G., Reeves, E. P., J Proteome Res 2014, 13, 3131-3143.

[23] Yin, H., Tan, Z., Wu, J., Zhu, J., Shedden, K. A., Marrero, J., Lubman, D. M., J Proteome Res 2015, $14,4876-4884$.

[24] Ceroni, A., Maass, K., Geyer, H., Geyer, R., Dell, A., Haslam, S. M., J Proteome Res 2008, 7, 16501659.

[25] Ajit Varki, R. D. C., Jeffrey D. Esko, Hudson H.Freeze, Pamela Stanley, Carolyn R. Bertozzi, Gerald W. Hart, Marilynn E.Etzler, Essentials of Glycobiology, Cold Spring Harbor Laboratory Press, New York 2008.

[26] Bones, S., Mittermayr, S., O'Donoghue, N., Guttman, A., Rudd, P. M., Anal Chem 2010, 82, 10208-10215.

[27] Murray, K., Boyd, R., Eberlin, M., Langley, G., Li, L. and Naito, Y., Pure and Applied Chemistry $2013,85,1515-1609$.

[28] Eliuk, S., Soltero, N., Remes, P., Senko, M., Zabrouskov, V. , $13^{\text {th }}$ Human Proteome Organization World Congress, Madrid, 2014, pp. 791-P.

[29] Patschull, A. O., Segu, L., Nyon, M. P., Lomas, D. A., Nobeli, I., Barrett, T. E., Gooptu, B., Acta crystallographica. Section F, Structural biology and crystallization communications 2011, 67, 1492 1497.

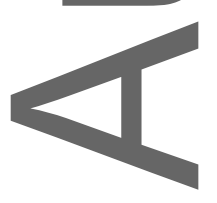

